\title{
An extremely large group index via electromagnetically induced transparency in metamaterials
}

\author{
A. Sayahian Jahromi \\ sayahian@gmail.com
}

M. Askari
Zarghan Branch, Islamic Azad university, Zarghan, Iran

Physics Department, College of Sciences, Salman Farsi University, Kazerun, Iran

We numerically demonstrate a planar design of a metamaterial exhibiting electromagnetically induced transparency at gigahertz frequencies. The design is based on the coupling of two oppositely oriented semi split-rings in the same plane. A very large transmission contrast of about $60 \mathrm{~dB}$ between the peak of the EIT window and the dips of the transmission gaps is calculated. An extremely large group index associated with the resonant response in the transmission window is demonstrated, rendering the design suitable for slow light applications. [DOI: http://dx.doi.org/10.2971/jeos.2014.14048]

Keywords: Metamaterials, electromagnetically induced transparency, slow light

\section{I NTRODUCTION}

Electromagnetically induced transparency (EIT) is a quantum mechanical effect observed in three level atomic media in which the absorption of a laser beam is significantly reduced in a narrow frequency band $[1,2]$. In a typical $\Lambda$ shape atomic media, there exist two atomic states with forbidden transition between them while both can be independently excited to the same final state. A probe laser beam with a frequency tuned to one of the transitions experiences a Lorentzian like absorption profile. In the presence of another coherent pump beam tuned to the other transition, a narrow transparency window is produced for the probe beam. This is due to the destructive interference between two excitation pathways of the excited state. While an anomalous dispersion is normally observed in a two-level resonance, there exists a strong normal dispersion at the resonance transparency frequency in these three-level atomic media, which can have unique practical applications such as slowing down of the light pulses and storage of data in matter [3]-[6].

EIT-like phenomena can also be observed in electromagnetic metamaterials (MMs) through the interaction of radiation with purely classical, subwavelength electromagnetic resonators with no pump source required [8]-[13]. Mimicking EIT with metamaterials have theoretically been shown [9]-[11], and experimentally been reported at microwave $[10,13,14]$, terahertz $[15,16]$, and optical frequencies $[17,18]$. In this class of metamaterials, transparency can be excited without quantum mechanical atomic states. This greatly reduces the difficulty in the experimental realization of EIT, and will hence bring us closer to its exciting applications.

In this letter, we introduce a metamaterial structure that exhibits an EIT-like behaviour in gigahertz $(\mathrm{GHz})$ frequencies when the structural symmetry is broken compared to the polarization direction of the incident electromagnetic wave. Our study shows that an extremely large group index of over 200 a)

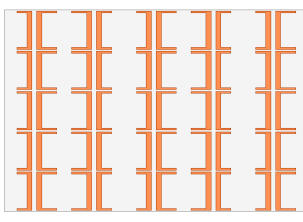

b)
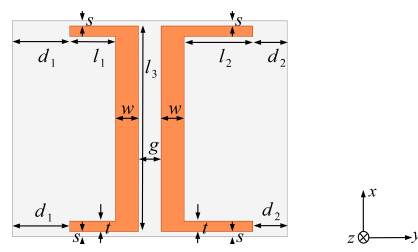

FIG. 1 (a) The planar structure proposed to exhibit EIT-like phenomena at microwave wavelengths. The structure is periodic in a way that every unit cell is the mirror image of its neighbour. (b) One unit cell of the structure with the parameters addressed in the text. The incident electromagnetic wave propagates along the $z$-direction with the electric field polarization along the $x$ - and the magnetic field polarization along the $y$ directions.

is achieved which is one of the highest values reported to date and which causes a large reduction factor for the group velocity.

\section{PROPOSED METAMATERIAL STRUCTURE}

As shown in Figure 1(b), the unit cell of the structure is composed of two metallic cutwires, with two metallic patches at the two ends of each cutwire, making two oppositely oriented semi split-rings (SSRs). In designing and simulating the planar metamaterial we use a commercial software package High-Frequency Structure Simulator (HFSS).

We have used two hollow waveguides with excitation ports, in front and behind the unit cell, to ensure the excitation and detection of linear polarized electromagnetic plane waves. Perfect electric and magnetic conductors were then set accordingly on the surrounding four walls of the waveguides to warrant the polarization of the electric field along the cutwires and the polarization of the magnetic field in the plane containing SSRs. 


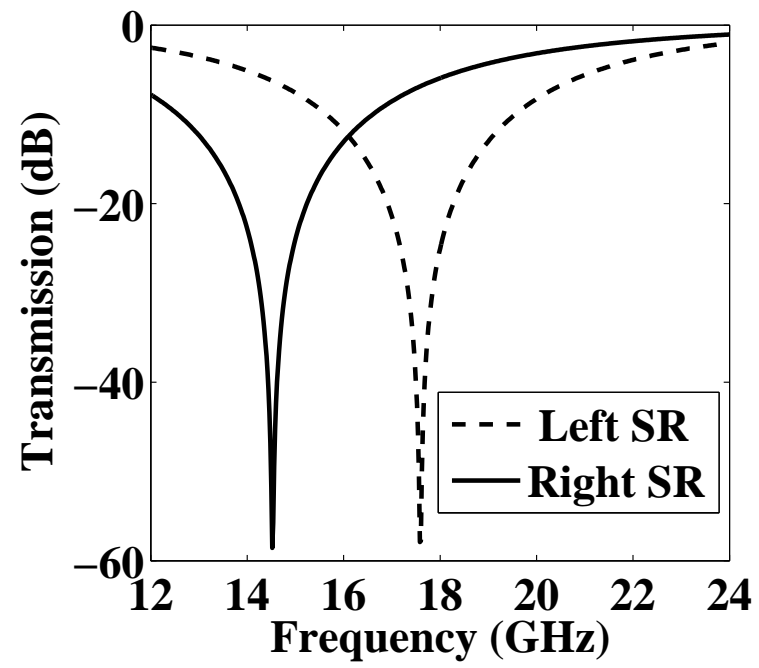

FIG. 2 The transmission from a metamaterial structure of Figure 1, with only left split ring present with $l_{1}=1 \mathrm{~mm}$ (the dashed curve) and with only right split ring present with $l_{2}=1.5 \mathrm{~mm}$ (the solid curve).

In the metamaterial structure, the metallic elements are deposited on a Rogers $\mathbf{5 8 8 0}$ substrate with a dielectric constant of 2.53 and a loss tangent 0.0009 . The thickness of the dielectric substrate is $254 \mu \mathrm{m}$ and it fills the whole unit cell in the lateral directions ( $x-y$ plane). For metallic elements, we use the lossy metal model with bulk conductivity $\sigma=5.8 \times 10^{7} \mathrm{~S} / \mathrm{m}$. The widths of metallic cut-wires are both $w=0.5 \mathrm{~mm}$ and the length of each is $l_{3}=4 \mathrm{~mm}$. The lengths of metallic patches are chosen so that $l_{1}=1 \mathrm{~mm}$ and $l_{2}=1.5 \mathrm{~mm}$, and their widths are $t=0.2 \mathrm{~mm}$. The gap between two cut-wires is $g=0.5 \mathrm{~mm}$, and the thicknesses of all metallic elements are $10 \mu \mathrm{m}$. The distances of the metal edges from the $x$-boundaries of the unit cell are all $s=0.1 \mathrm{~mm}$ and the distances of the metallic patches ends from the left and the right y-boundaries are respectively $d_{1}=1.25 \mathrm{~mm}$ and $d_{2}=0.75 \mathrm{~mm}$. The unit cell size along the $x$-and $y$-directions are, respectively, $4.2 \mathrm{~mm}$ and $6 \mathrm{~mm}$.

\section{NUMERICAL CALCULATIONS AND DISCUSSION}

We consider a plane electromagnetic (EM) wave, incident normal to the surface of the structure, i.e., the propagation direction of the incident wave is taken to be along the $z$-direction and perpendicular to the $x-y$ plane (see Figure $1(b)$ ). The polarization of the incident wave is such that its electric field component is along cut-wires ( $x$-direction) and the magnetic field component is in the plane of the wires (y-direction). Since, the magnetic field has no component normal to the plane of the SSRs, this choice of the polarization inhibits a magnetic resonance in SSRs directly induced by the magnetic field component of the wave. However, each SSR can be modeled as a LC resonator. The inductance is provided by the loop of the SSR and the capacitance is provided by the gap between the arms of the SSR. The LC resonance of each SSR is induced when the polarization of the electric field is along the cutwire. Since the electric field component of the incident wave is along the cut-wires, an LC resonance can be induced in the SSRs [19]. In case of a LC resonance in each SSR, two equal

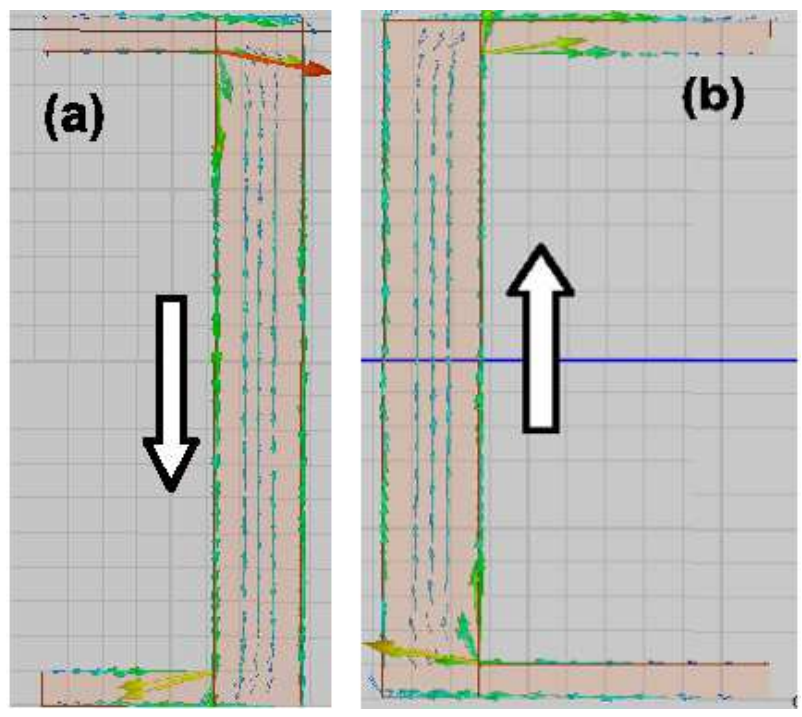

FIG. 3 (a) The surface current density for the metamaterial structure of Figure 1(a) with only left split ring present with $l_{1}=1 \mathrm{~mm}$ at $17.6 \mathrm{CHz}$, and (b) with only right split ring present with $l_{2}=1.5 \mathrm{~mm}$ at $14.9 \mathrm{GHz}$.

antiparallel currents in the end patches result in a vanishing electric dipole moment, however, the induced current in the cutwire results in a net electric dipole moment that provides a considerable scattering loss. If the lengths of the patches for the left and the right SSRs are different, the LC resonance frequencies of the SSRs will also be different. When the two SSRs are brought close to each other (as in Figure 1), a so-called trapped mode is excited which induces two equal antiparallel currents in cutwires, resulting in a sharp resonant feature splitting a very wide transmission stop band.

In order to find out the role of each SSR in the resulting composite structure, we first prefer to study each SSR element separately. First, we consider a structure with only the left SSR, with $l_{1}=1 \mathrm{~mm}$. As shown in Figure 2, the transmission spectrum for this element alone, in the absence of the right SSR, has a dip at $17.6 \mathrm{GHz}$ representing a resonance at this frequency. To find out the underlying physics, we simulate the surface current distribution on the surface of this SSR at 17.6 GHz. As can be seen in Figure 3(a), the current distribution shows an induced electric dipole moment along the cutwire which causes a scattering loss and a resonance feature at 17.6 GHz in the transmission spectrum. The dipole moments in the patches at the two ends of the cut-wire are in opposite directions, resulting in no net electric dipole moment.

Eliminating the left SSR, in the next step, only the right SSR with $l_{2}=1.5 \mathrm{~mm}$ is taken into account. Figure 2 also shows the transmission spectrum for the structure composed of this element alone. As can be seen in the Figure, there exists a stop band in the transmission spectrum, at $14.92 \mathrm{GHz}$. The results of the simulation of the surface current distribution at this frequency are shown in Figure 3(b). The current distribution confirmed existence of a net electric dipole moment induced in the cut wire resulting in a large scattering loss and therefore a stop band at $14.92 \mathrm{GHz}$.

We now bring these two elements together as depicted in Figure 1 , with $l_{1}=1 \mathrm{~mm}$ and $l_{2}=1.5 \mathrm{~mm}$. The transmission 

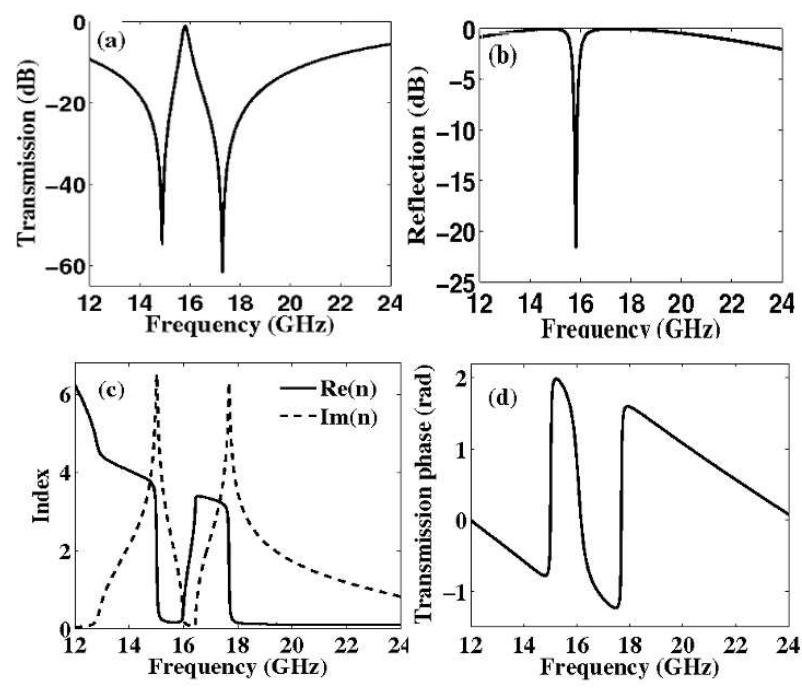

FIG. 4 (a) The transmission and (b) the reflection from the metamaterial structure of Figure 1 in the composite form, with $l_{1}=1 \mathrm{~mm}$ and $l_{2}=1.5 \mathrm{~mm}$. (c) The real part and the imaginary part of the retrieved refractive index for the proposed structure. (d) The transmission phase.

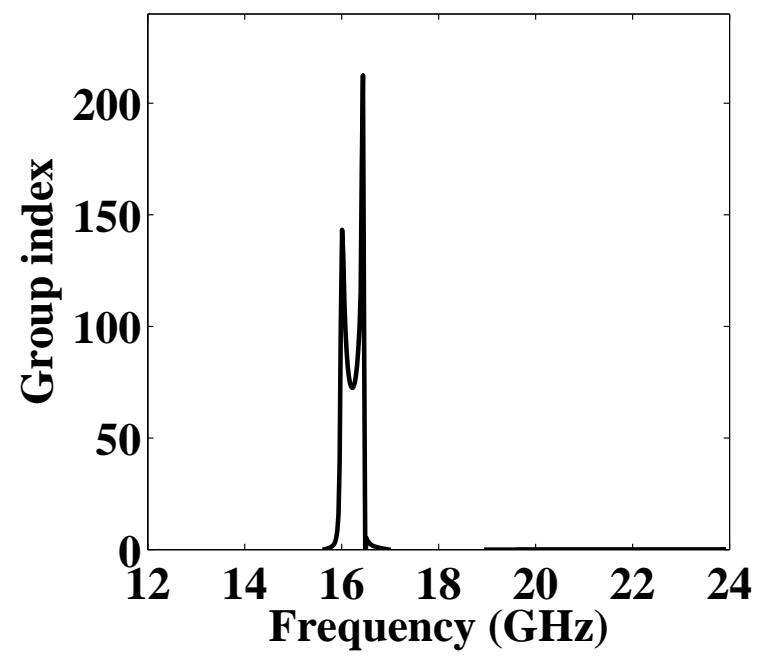

FIC. 5 The group index obtained from the scattering parameters. At transparency frequencies a group index of more than 200 is measured.

and reflection spectra for this composite structure are shown in Figures 4(a) and 4(b). As can be seen from the transmission spectrum, there still exist two stop bands near $14.9 \mathrm{GHz}$ and $17.3 \mathrm{GHz}$, respectively corresponding to stop bands for the structures with only the right SR present and only the left SR present. However, in the present case, a narrow transparency band appears between the two stop bands. As can be seen in Figure 4(d), the transmission window is accompanied by a very sharp normal phase dispersion that renders the response of the structure a metamaterial analogue of EIT $[10,9]$. There exists a very large transmission contrast of about $60 \mathrm{~dB}$ between the peak of the EIT window and the dips of the transmission gaps which is very large compared to the metamaterial structures reported to date [21, 14]. Using the parameter retrieval procedure [20] for a metamaterial of thickness $2.7 \mathrm{~mm}$ along the $z$-direction, we obtain the refractive index $n$. Figure 4 (c) shows that the imaginary part of the refractive index represents a dip corresponding to a low loss

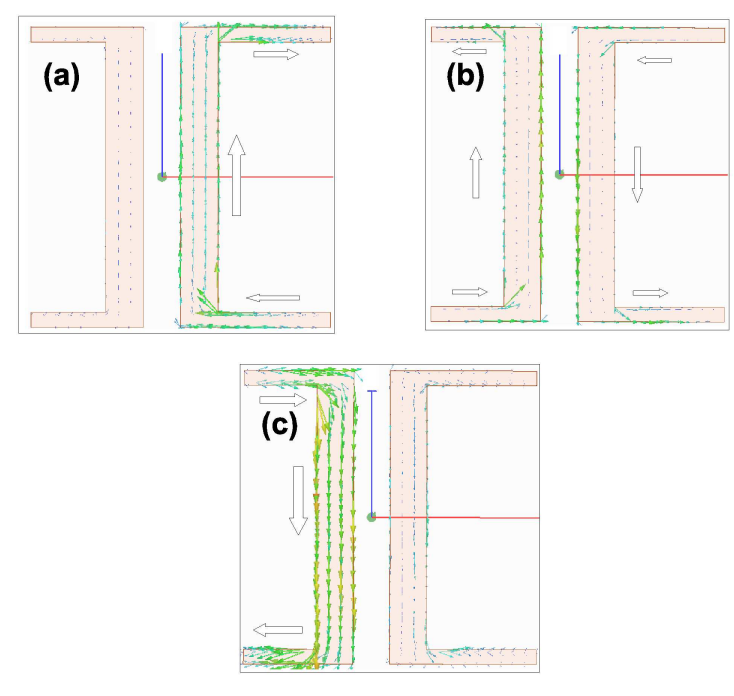

FIG. 6 (a)The surface current density for the metamaterial structure of Figure 1 with $l_{1}=1 \mathrm{~mm}$ and $l_{2}=1.5 \mathrm{~mm}$ at (a) $14.9 \mathrm{GHz}$, and (b) $15.8 \mathrm{GHz}$ and (c) $17.3 \mathrm{GHz}$.

in the transparency window. Simultaneously, the real part of $n$ shows a strong dispersion which results in a significantly increased group index in the transparency window. The group index defined by $n_{g}=n+\omega d n / d \omega$, was also calculated using the retrieved refractive index. The result is shown in Figure 5. The group index reaches a value over 200 in the transparency window which is a significantly large value and among the largest values for the group index reported so far [21]. This large value of the group index results in a large reduction factor for the group velocity. We also calculate the group delay defined by $t_{g}=-d \phi(\omega) / d \omega$ in which $\phi$ is the transmission phase. We obtain a value $140 \mathrm{~ns}$ for the group delay at the transparency frequency.

Simulations of surface current distributions are represented in Figure 6. As can be seen in Figure 6(a), at the stop band frequency $14.9 \mathrm{GHz}$, only the right SSR couples with the incident electromagnetic wave and while an electric dipole resonance is induced in the right SSR, there is no current distribution in the left SSR. The electric dipole induced in the cutwire of the right SSR provides a radiation resistance resulting in a stop band in the transmission spectrum. Figure 6(c) shows current distributions for the other stop band at 17.3 GHz. At this frequency, only an electric dipole is excited in the left SSR with no resonance in the right SSR. Figure 6(b) reveals that at the transparency frequency $15.8 \mathrm{GHz}$, electric dipole resonances in the both SSRs are simultaneously excited inducing two equal antiparallel currents along the cutwires which means opposite directions of induced electric dipoles. Therefore, the net electric dipole moment of the structure vanishes resulting in a vanishing radiation resistance and therefore resulting in a transparency.

\section{CONCLUSION}

In conclusion, we introduce a structure composed of two asymmetric semi split-rings. Whereas structures composed of each SSR alone represent band gaps, each at an individual frequency, the structure composed of both SSRs represents a transparency window with a high transmission at a frequency 
at which the structure with each SSR alone is opaque. A very large transmission contrast of about $60 \mathrm{~dB}$ between the peak of the EIT window and the dips of the transmission gaps is observed which is very large compared to the corresponding values for the metamaterial structures reported to date. In the transparency window, the group index reaches a value over 200 which is significantly large and among the largest values for the group indices reported so far.

\section{References}

[1] A. B. Matsko, 0. Kocharovskaya, Y. Rostovtsev, G. R. Welch, A. S. Zibrov, and M. 0. Scully, "Slow, ultraslow, stored, and frozen light," Adv. At. Mol. Opt. Phys. 46, 191-242 (2001).

[2] M. Fleischhauer, A. Imamoglu, and J. P. Marangos, "Electromagnetically induced transparency: Optics in coherent media," Rev. Mod. Phys. 77, 633-673 (2005).

[3] L.V. Hau, S. E. Harris, Z. Dutton, and C. H. Behroozi, "Light speed reduction to 17 metres per second in an ultracold atomic gas," Nature 397, 594-598 (1999).

[4] M. Fleischhauer, and M. D. Lukin, "Dark-State Polaritons in Electromagnetically Induced Transparency," Phys. Rev. Lett. 84, 5094-5097 (2000).

[5] C. Liu, Z. Dutton, C. H. Behroozi, and L.V. Hau, "Observation of coherent optical information storage in an atomic medium using halted light pulses," Nature 409, 490-493 (2001).

[6] M. Bajcsy, A. S. Zibrov, and M. D. Lukin, "Stationary pulses of light in an atomic medium," Nature 426, 638-641 (2003).

[7] C. L. Garrido Alzar, M.A.G. Martinez, and P. Nussenzveig, "Classical analog of electromagnetically induced transparency," Am. J. Phys. 70, 37-41 (2002).

[8] V. A. Fedotov, M. Rose, S. L. Prosvirnin, N. Papasimakis, and N. I. Zheludev, "Sharp Trapped-Mode Resonances in Planar Metamaterials with a Broken Structural Symmetry," Phys. Rev. Lett. 99, 147401 (2007).

[9] S. Zhang, D. A. Genov, Y. Wang, M. Liu, and X. Zhang, "PlasmonInduced Transparency in Metamaterials," Phys. Rev. Lett. 101, 047401 (2008).

[10] N. Papasimakis, V. A. Fedotov, N. I. Zheludev, and S. L. Prosvirnin, "Metamaterial Analog of Electromagnetically Induced Transparency," Phys. Rev. Lett. 101, 253903 (2008).
[11] P. Tassin, L. Zhang, Th. Koschny, E. N. Economou, and C. M. Soukoulis, "Low-Loss Metamaterials Based on Classical Electromagnetically Induced Transparency," Phys. Rev. Lett. 102, 053901 (2009).

[12] N. Papasimakis, Y. H. Fu, V. A. Fedotov, S. L. Prosvirnin, D. P. Tsai, and N. I. Zheludev, "Metamaterial with polarization and direction insensitive resonant transmission response mimicking electromagnetically induced transparency," Appl. Phys. Lett. 94, 211902 (2009).

[13] N. Niakan, M. Askari, and A. Zakery, "High Q-factor and large group delay at microwave wavelengths via electromagnetically induced transparency in metamaterials," J. Opt. Soc. Am. B. 29, 2329-2333 (2012).

[14] C. Kurter, P. Tassin, L. Zhang, T. Koschny, A. P. Zhuravel, A. V. Ustinov, S. M. Anlage, et al., "Classical Analogue of Electromagnetically Induced Transparency with a Metal-Superconductor Hybrid Metamaterial," Phys. Rev. Lett. 107, 043901 (2011).

[15] R. Singh, C. Rockstuhl, F. Lederer, and W. Zhang, "Coupling between a dark and a bright eigenmode in a terahertz metamaterial," Phys. Rev. B. 79, 085111 (2009).

[16] S. Y. Chiam, R. Singh, C. Rockstuhl, F. Lederer, W. Zhang, and A. A. Bettiol, "Analogue of electromagnetically induced transparency in a terahertz metamaterial," Phys. Rev. B. 80, 153103 (2009).

[17] N. Liu, L. Langguth, T. Weiss, J. Kästel, M. Fleischhauer, T. Pfau, and $H$. Cissen, "Plasmonic analogue of electromagnetically induced transparency at the Drude damping limit," Nat. Mater. 8, 758-762 (2009).

[18] N. Verellen, Y. Sonnefraud, H. Sobhani, F. Hao, V. V. Moshchalkov, P. Van Dorpe, P. Nordlander, and S. A. Maier, "Fano Resonances in Individual Coherent Plasmonic Nanocavities," Nano Lett. 9, 1663-1667 (2009).

[19] N. Katsarakis, T. Koschny, M. Kafesaki, E. N. Economou, and C. M. Soukoulis, "Electric coupling to the magnetic resonance of split ring resonators," Appl. Phys. Lett. 84, 2943-2945 (2004).

[20] D. R. Smith, D. C. Vier, T. Koschny, and C. M. Soukoulis, "Electromagnetic parameter retrieval from inhomogeneous metamaterials," Phys. Rev. E 71, 036617 (2005).

[21] L. Zhang, P. Tassin, T. Koschny, C. Kurter, S. M. Anlage, and C. M. Soukoulis, "Large group delay in a microwave metamaterial analog of electromagnetically induced transparency," Appl. Phys. Lett. 97, 241904 (2010). 